Journal of Applied AnALysis

Vol. 8, No. 1 (2002), pp. 83-110

\title{
LINEAR FREDHOLM INTEGRAL EQUATIONS AND THE INTEGRAL OF KURZWEIL
}

\author{
M. FEDERSON and R. BIANCONI \\ Received September 14, 2000 and, in revised form, March 27, 2001
}

\begin{abstract}
We apply the Kurzweil-Henstock integral setting to prove a Fredholm Alternative-type result for the integral equation

$$
x(t)-{ }^{K} \int_{[a, b]} \alpha(t, s) x(s) d s=f(t), \quad t \in[a, b],
$$

where $x$ and $f$ are Kurzweil integrable functions (possibly highly oscillating) defined on a compact interval $[a, b]$ of the real line with values on Banach spaces. An application is given.
\end{abstract}

\section{Introduction}

The Kurzweil-Henstock integral setting has been shown to be useful not only in giving simple treatment to sophisticated matters (e.g.: [26], [27]), but also in sharpenning many classical results (e.g.: [3], [4]). Its utility can also be verified in different approaches to Integral Equations (e.g.: [9], [30], [31]).

2000 Mathematics Subject Classification. 45A05, 45B05, 26A39.

Key words and phrases. Linear integral equations, Fredholm Alternative, KurzweilHenstock integrals.

ISSN 1425-6908（C) Heldermann Verlag. 
When we consider real-valued functions, the integrals defined by Kurzweil ([21]) and independently by Henstock ([13]) coincide. The KurzweilHenstock integral generalizes the integrals of Riemann, Lebesgue and Newton and has good convergence properties (e.g.: [12], [14], [22], [23], [25], [28]).

However, in a general Banach space-valued context, it may happen that the space of Henstock integrable functions is properly contained in the space of Kurzweil integrable ones. In this context, one can find an abstract Riemann integral which is not Henstock integrable ([9], Example 1.1). Moreover, while the abstract Fundamental Theorem of Calculus holds for the Henstock integral ([6]), it may not be valid for the Kurzweil integral because if the Kurzweil integral of a function is zero, then it is not necessarily true that the function itself is zero almost everywhere in Lebesgue's sense ([9], Example 1.1). On the other hand, we can establish abstract Monotone Convergence Theorems for the Kurzweil integral but not for the integral of Henstock ([8], Example 17).

In this paper, we deal with Banach space-valued functions. Although our results concern only the Kurzweil integral, we present both the abstract integrals of Kurzweil and of Henstock in view of their differences and uses in the proofs. It is worth mentioning however that the main results also hold if we replace the Kurzweil integral by that of Henstock.

Despite being non-complete, the associated spaces of all equivalence classes of either Kurzweil or Henstock integrable functions, equipped with the Alexiewicz norm, have good functional analytic properties ([1], [18], [11]). Because of these non-completeness, usual Fixed Point Theorems can not be applied in order to obtain results on existence or uniqueness of a solution of the linear Fredholm integral equation

$$
x(t)-K \int_{[a, b]} \alpha(t, s) x(s) d s=f(t), \quad t \in[a, b],
$$

and, in particular, of the linear Volterra integral equation

$$
x(t)-K \int_{[a, t]} \alpha(t, s) x(s) d s=f(t), \quad t \in[a, b],
$$

where $x$ and $f$ are Kurzweil or Henstock integrable functions, $[a, b]$ is a compact interval of the real line $\mathbb{R}$.

On the other hand, the ideas due to C. S. Hönig ([19]; see also [10]) of

- transforming equations (1) and (2) into integral equations with respect to the Riemann-Stieltjes integral and of

- using isometries to obtain results on existence or uniqueness of a solution of such Stieltjes equations, 
put aside the problem of non-completeness. Suppose $\alpha$ fulfills certain properties. Hönig's result for equation (2) establishes the existence and uniqueness of a solution:

(A) given a Kurzweil integrable function $f:[a, b] \rightarrow X, X$ a Banach space, equation (2) admits one and only one Kurzweil integrable solution $x_{f}$ with resolvent given by the Neumann series (see [19] or [10], Corollary 28).

Hönig's result for equation (1) gives an explicit solution provided it is known beforehand that there exists a solution and it is unique:

(B) if $f:[a, b] \rightarrow X, X$ a Banach space, is a Kurzweil integrable function such that there is one and only one Kurzweil integrable solution $x_{f}$ of (1), then $f \mapsto x_{f}$ is bicontinuous and the resolvent of (1) has similar integral representation, that is

$$
x_{f}(t)=f(t)-K \int_{[a, b]} \phi(t, s) f(s) d s, \quad t \in[a, b],
$$

where $\phi$ satisfies similar conditions required for $\alpha$.

In the present notes, we give conditions for the existence of a solution of (1). In order to do this, we answer affirmatively to a conjecture of Hönig which goes back to 1978. This will imply a Fredholm Alternative-type result for a Volterra-Stieltjes integral equation. Finally, assuming that $\alpha$ satisfies adequate conditions, we apply Hönig's ideas mentioned above to obtain a Fredholm Alternative-type result for equation (1):

(C) given a Kurzweil integrable function $f:[a, b] \rightarrow X, X$ a Banach space, either equation (1) has one and only one solution with resolvent having similar integral representation, or the corresponding homogeneous equation

$$
u(t)-K \int_{[a, b]} \alpha(t, s) u(s) d s=0, \quad t \in[a, b],
$$

has non-trivial solutions. In this case, further conditions are assumed so that (1) admits a solution.

This paper is organized as follows. Section 2 is devoted to definitions and basic properties of the abstract Riemann-Stieltjes integrals and the presentation of a Fredholm Alternative-type result for Riemann-Stieltjes integral equations. In Section 3, we present the abstract integrals of Kurzweil and of Henstock. Also in Section 3 we give some auxiliary results and we present the Fredholm Alternative-type result for linear integral equations with respect to the Kurzweil integral. An application to boundary value problems can be found in Section 4. 


\section{The Riemann-Stieltjes integrals}

\subsection{Definitions and terminology.}

A bilinear triple (we write $B T$ ) is a set of three vector spaces $E, F$ and $G$, where $F$ and $G$ are Banach spaces with a bilinear mapping $\mathcal{B}: E \times F \rightarrow$ $G$. For $x \in E$ and $y \in F$, we write $x y=\mathcal{B}(x, y)$ and we denote the $B T$ by $(E, F, G)_{\mathcal{B}}$ or simply by $(E, F, G)$. A topological $B T$ is a $B T(E, F, G)$ where $E$ is also a normed space and $\mathcal{B}$ is continuous. We suppose that $\|\mathcal{B}\| \leq 1$.

If $E$ and $F$ are normed spaces, then we denote by $L(E, F)$ the space of all linear continuous functions from $E$ to $F$. We write $E^{\prime}=L(E, \mathbb{R})$ and $L(E)=L(E, E)$, where $\mathbb{R}$ denotes the real line.

Throughout this paper, $X, Y$ and $Z$ always denote Banach spaces.

Example 2.1. As an example of a $B T$ we can consider $E=L(X, Y)$, $F=L(Z, X), G=L(Z, Y)$ and $\mathcal{B}(v, u)=v \circ u$. In particular, when $Z=\mathbb{R}$, we have $E=L(X, Y), F=X, G=Y$ and $\mathcal{B}(u, x)=u(x)$; when $X=\mathbb{R}$, we have $E=Y, F=Y^{\prime}, G=\mathbb{R}$ and $\mathcal{B}\left(y, y^{\prime}\right)=\left\langle y, y^{\prime}\right\rangle$; when $X=Z=\mathbb{R}$, we have $E=G=Y, F=\mathbb{R}$ and $\mathcal{B}(y, \lambda)=\lambda y$.

Let $[a, b]$ be a compact interval of $\mathbb{R}$. Any finite set of closed nonoverlapping subintervals $\left[t_{i-1}, t_{i}\right]$ of $[a, b]$ such that the union of all intervals $\left[t_{i-1}, t_{i}\right]$ equals $[a, b]$ is called a division of $[a, b]$. In this case we write $d=\left(t_{i}\right) \in D_{[a, b]}$, where $D_{[a, b]}$ denotes the set of all divisions of $[a, b]$. By $|d|$ we mean the number of subintervals in which $[a, b]$ is divided through a given $d \in D_{[a, b]}$.

A tagged division of $[a, b]$ is any set of pairs $\left(\xi_{i}, t_{i}\right)$ such that $\left(t_{i}\right) \in D_{[a, b]}$ and $\xi_{i} \in\left[t_{i-1}, t_{i}\right]$ for every $i$. In this case we write $d=\left(\xi_{i}, t_{i}\right) \in T D_{[a, b]}$, where $T D_{[a, b]}$ denotes the set of all tagged divisions of $[a, b]$. Any subset of a tagged division of $[a, b]$ is a tagged partial division of $[a, b]$ and, in this case, we write $d \in T P D_{[a, b]}$.

A gauge of a set $E \subset[a, b]$ is any function $\delta: E \rightarrow] 0, \infty[$. Given a gauge $\delta$ of $[a, b]$, we say that $d=\left(\xi_{i}, t_{i}\right) \in T P D_{[a, b]}$ is $\delta$-fine, if $\left[t_{i-1}, t_{i}\right] \subset$ $\left\{t \in[a, b] ;\left|t-\xi_{i}\right|<\delta\left(\xi_{i}\right)\right\}$ for every $i$.

We now will define the Riemann-Stieltjes integrals by means of tagged divisions $d=\left(\xi_{i}, t_{i}\right)$ of $[a, b]$ and constant gauges $\delta$ (i.e., there is a $\delta_{0}>0$ such that $\delta(\xi)=\delta_{0}$ for every $\left.\xi \in[a, b]\right)$.

Let $(E, F, G)$ be a $B T$. Any function $\alpha:[a, b] \rightarrow E$ is said to be Riemann integrable with respect to a function $f:[a, b] \rightarrow F$ if there exists an $I \in G$ such that for every $\varepsilon>0$, there is a constant gauge $\delta$ of $[a, b]$ such that for 
every $\delta$-fine $d=\left(\xi_{i}, t_{i}\right) \in T D_{[a, b]}$,

$$
\left\|\sum_{i=1}^{|d|}\left[\alpha\left(t_{i}\right)-\alpha\left(t_{i-1}\right)\right] f\left(\xi_{i}\right)-I\right\|<\varepsilon .
$$

We write $I=\int_{[a, b]} d \alpha(t) f(t)$ in this case. By $R_{f}([a, b], E)$ we mean the space of all functions $\alpha:[a, b] \rightarrow E$ which are Riemann integrable with respect to $f:[a, b] \rightarrow F$.

Analogously we define the Riemann integral of $f:[a, b] \rightarrow F$ with respect to $\alpha:[a, b] \rightarrow E$ when it exists. We say that $f:[a, b] \rightarrow F$ is Riemann integrable with respect to $\alpha:[a, b] \rightarrow E$ if there exists an $I \in G$ such that for every $\varepsilon>0$, there is a constant gauge $\delta$ of $[a, b]$ such that for every $\delta$-fine $d=\left(\xi_{i}, t_{i}\right) \in T D_{[a, b]}$,

$$
\left\|\sum_{i=1}^{|d|} \alpha\left(\xi_{i}\right)\left[f\left(t_{i}\right)-f\left(t_{i-1}\right)\right]-I\right\|<\varepsilon .
$$

Then $R^{\alpha}([a, b], F)$ denotes the space of all functions $f:[a, b] \rightarrow F$ which are Riemann integrable with respect to a given $\alpha:[a, b] \rightarrow E$ with integral $I=\int_{[a, b]} \alpha(t) d f(t)$.

The integrals $\int_{[a, b]} d \alpha(t) f(t)$ and $\int_{[a, b]} \alpha(t) d f(t)$ defined above are known as Riemann-Stieltjes integrals.

Given Banach spaces $X$ and $Y$ and $u \in L(X, Y)$, we denote by $u^{*} \in$ $L\left(Y^{\prime}, X^{\prime}\right)$ the transposed operator defined by $\left\langle x, u^{*}\left(y^{\prime}\right)\right\rangle=\left\langle u(x), y^{\prime}\right\rangle$, where $x \in X$ and $y^{\prime} \in Y^{\prime}$. Then, for $y^{\prime} \in Y^{\prime}$ and $u \in L(X, Y)$, we have

$$
y^{\prime} \circ u=u^{*} y^{\prime},
$$

since $\left(y^{\prime} \circ u\right)(x)=\left\langle y^{\prime}, u x\right\rangle=\left\langle u^{*} y^{\prime}, x\right\rangle$ for every $x \in X$. Thus, when we consider the $B T(E, F, G)$ with $E=Y^{\prime}=L(Y, \mathbb{R}), F=L(X, Y), G=$ $X^{\prime}=L(X, \mathbb{R})$ and $\mathcal{B}\left(y^{\prime}, u\right)=y^{\prime} \circ u, y^{\prime} \in Y^{\prime}$ and $u \in L(X, Y)$, we use the identification

$$
\int_{[c, d]} d y(t) \circ K(t, s)=\int_{[c, d]} K(t, s)^{*} d y(t),
$$

where $y:[a, b] \rightarrow Y^{\prime}, K:[c, d] \times[a, b] \rightarrow L(X, Y)$ and $K(t, s)^{*}$ is the transposed operator of $K(t, s) \in L(X, Y)$. 
2.2. Functions of bounded $\mathcal{B}$-variation, of bounded semi-variation and of bounded variation.

Given a $B T(E, F, G)_{\mathcal{B}}$ and $\alpha:[a, b] \rightarrow E$, for every division $d=\left(t_{i}\right) \in$ $D_{[a, b]}$ we define

$$
S B_{d}(\alpha)=S B_{[a, b], d}(\alpha)=\sup \left\{\left\|\sum_{i=1}^{|d|}\left[\alpha\left(t_{i}\right)-\alpha\left(t_{i-1}\right)\right] y_{i}\right\| ; y_{i} \in F,\left\|y_{i}\right\| \leq 1\right\}
$$

and

$$
S B(\alpha)=S B_{[a, b]}(\alpha)=\sup \left\{S B_{d}(\alpha) ; d \in D_{[a, b]}\right\} .
$$

Then $S B(\alpha)$ is the $\mathcal{B}$-variation of $\alpha$ on $[a, b]$. We say that $\alpha$ is a function of bounded $\mathcal{B}$-variation whenever $S B(\alpha)<\infty$. When this is the case, we write $\alpha \in S B([a, b], E)$.

The following properties are not difficult to prove:

- $S B([a, b], E)$ is a vector space and

$$
\alpha \in S B([a, b], E) \mapsto S B(\alpha) \in \mathbb{R}_{+}
$$

is a seminorm;

- for $\alpha \in S B([a, b], E)$, the function

$$
t \in[a, b] \mapsto S B_{[a, t]}(\alpha) \in \mathbb{R}_{+}
$$

is monotonically increasing;

- for $\alpha \in S B([a, b], E)$, we have

$$
S B_{[a, b]}(\alpha) \leq S B_{[a, c]}(\alpha)+S B_{[c, b]}(\alpha)
$$

for every $c \in] a, b[$.

We define

$$
S B_{c}([a, b], E)=\{\alpha \in S B([a, b], E) ; \alpha(c)=0\}, \quad c \in[a, b] .
$$

Example 2.2. Given Banach spaces $X$ and $Y$, consider the $B T(L(X, Y)$, $X, Y)$. Then we write $S V(\alpha)$ instead of $S B(\alpha)$ and $S V([a, b], L(X, Y))$ instead of $S B([a, b], L(X, Y))$. The elements of $S V([a, b], L(X, Y))$ are called functions of bounded semi-variation.

Given a function $f:[a, b] \rightarrow E, E$ a normed space, and $d=\left(t_{i}\right) \in D_{[a, b]}$, we define

$$
V_{d}(f)=V_{d,[a, b]}(f)=\sum_{i=1}^{|d|}\left\|f\left(t_{i}\right)-f\left(t_{i-1}\right)\right\|
$$

and the variation of $f$ is given by

$$
V(f)=V_{[a, b]}(f)=\sup \left\{V_{d}(f) ; d \in D_{[a, b]}\right\} .
$$


If $V(f)<\infty$, then $f$ is called a function of bounded variation. In this case, we write $f \in B V([a, b], E)$.

When we consider the $B T\left(Y, Y^{\prime}, \mathbb{R}\right)$, we write $B V(\alpha)$ and $B V\left([a, b], Y^{\prime}\right)$ instead of $S B(\alpha)$ and $S B\left([a, b], Y^{\prime}\right)$ respectively ([16, p. 22]). The inclusion $B V([a, b], L(E, F)) \subset S V([a, b], L(E, F))$ holds. Moreover, $S V([a, b]$, $L(E, \mathbb{R}))=B V\left([a, b], E^{\prime}\right)$.

Let $X$ and $Y$ be Banach spaces. Given $c \in[a, b]$, we define the spaces

$$
\begin{gathered}
B V_{c}([a, b], X)=\{f \in B V([a, b], X) ; f(c)=0\}, \\
B V_{c}^{+}([a, b], X)=\left\{f \in B V_{c}([a, b], X) ; f \text { is right continuous }\right\}, \\
S V_{c}([a, b], L(X, Y))=\{\alpha \in S V([a, b], L(X, Y)) ; \alpha(c)=0\}
\end{gathered}
$$

all of which are complete when endowed with the norm given by the variation $V(f)$ (in the first two cases) and the semi-variation $S V(\alpha)$ (in the last case).

The reader may want to consult [16] for more details and properties of all these spaces.

\subsection{Preliminary results.}

Let $E$ be a normed space. By $C([a, b], E)$ we mean the space of all continuous functions from $[a, b]$ to $E$ endowed with the usual supremum norm $\|\cdot\|_{\infty}$. We define

$$
C_{a}([a, b], E)=\{f \in C([a, b], E) ; f(a)=0\} .
$$

The next result is well-known. It gives the Integration by Parts Formula for the Riemann-Stieltjes integrals. For a proof of it, see [16].

Theorem 2.1. Let $(E, F, G)$ be a BT. If either $\alpha \in S B([a, b], E)$ and $f \in C([a, b], F)$, or $\alpha \in C([a, b], E)$ and $f \in B V([a, b], F)$, then $\alpha \in$ $R_{f}([a, b], E), f \in R^{\alpha}([a, b], F)$ and the Integration by Parts Formula

$$
\int_{[a, b]} d \alpha(t) f(t)=\alpha(b) f(b)-\alpha(a) f(a)-\int_{[a, b]} \alpha(t) d f(t)
$$

holds.

The next result is the Riemann-Stieltjes integral version of the well-known Saks-Henstock Lemma (Lemma 3.2 in the sequence). Its proof follows the standard steps (see for instance [29, Proposition 16]).

Lemma 2.1 (Saks-Henstock Lemma). If $\varepsilon>0$ and $\delta$ is a constant gauge of $[a, b]$ such that for every $\delta$-fine $d=\left(\xi_{i}, t_{i}\right) \in T D_{[a, b]}$,

$$
\left\|\int_{[a, b]} \alpha(t) d f(t)-\sum_{i=1}^{|d|} \alpha\left(\xi_{i}\right)\left[f\left(t_{i}\right)-f\left(t_{i-1}\right)\right]\right\|<\varepsilon,
$$


then for every $\delta$-fine $d=\left(\zeta_{j}, s_{j}\right) \in T P D_{[a, b]}$,

$$
\left\|\sum_{j=1}^{|d|}\left\{\int_{\left[s_{j-1}, s_{j}\right]} \alpha(t) d f(t)-\alpha\left(\zeta_{j}\right)\left[f\left(s_{j}\right)-f\left(s_{j-1}\right)\right]\right\}\right\|<\varepsilon .
$$

Remark 2.1. An analogous result holds for $\int_{[a, b]} d \alpha(t) f(t)$ as well.

A proof of the next result can be carried out by following the ideas of [7], Corollary 8 using Theorem 2.1 and Lemma 2.1.

Theorem 2.2. Suppose $\alpha \in S V([a, b], L(X, Y)), f \in C([a, b], Z), \beta \in$ $R_{f}([a, b], L(Z, X))$ and $\gamma(t)=\int_{[a, t]} \beta(s) d f(s)$, for every $t \in[a, b]$. Then $\alpha(\cdot) \beta(\cdot) \in R_{f}([a, b], L(Z, Y))$ and

$$
\int_{[a, b]} \alpha(t) \beta(t) d f(t)=\int_{[a, b]} \alpha(t) d \gamma(t) .
$$

A function $\alpha:[a, b] \rightarrow L(X, Y)$ is said to be weakly continuous (we write $\left.\alpha \in C^{\sigma}([a, b], L(X, Y))\right)$ if for every $x \in X$ the function

$$
\alpha(\cdot) x: t \in[a, b] \mapsto \alpha(t) x \in Y
$$

is continuous.

Given a function $K:(t, s) \in[c, d] \times[a, b] \mapsto K(t, s) \in L(X, Y)$, we write $K^{t}(s)=K_{s}(t)=K(t, s)$. Suppose $K$ is weakly continuous as a function of the first variable and uniformly of bounded semi-variation as a function of the second variable, that is,

- given $s \in[a, b], K_{s}$ is weakly continuous (i.e., the function $K_{s}(\cdot) x$ : $t \in[a, b] \mapsto K_{s}(t) x \in Y$ is continuous for every $\left.x \in X\right)$ and

- given $t \in[c, d], K^{t}$ is uniformly of bounded semi-variation (i.e., $K^{t}$ belongs to $S V([a, b], L(X, Y))$ and $S V^{u}(K)=\sup \left\{S V\left(K^{t}\right)\right.$; $t \in[c, d]\}<\infty)$.

Then we write $K \in C^{\sigma} \times(S V)^{u}([c, d] \times[a, b], L(X, Y))$. If in addition $K^{t}(a)=0$ for every $t \in[c, d]$, then $K \in C^{\sigma} \times\left(S V_{a}\right)^{u}([c, d] \times[a, b], L(X, Y))$. When functions of bounded variation are considered instead of functions of bounded semi-variation, we replace $C^{\sigma} \times(S V)^{u}([c, d] \times[a, b], L(X, Y))$ by $C^{\sigma} \times(B V)^{u}([c, d] \times[a, b], L(X, Y))$ and $C^{\sigma} \times\left(S V_{a}\right)^{u}([c, d] \times[a, b], L(X, Y))$ by $C^{\sigma} \times\left(B V_{a}\right)^{u}([c, d] \times[a, b], L(X, Y))$.

We use the notation $\int_{[a, b]} d_{s} K(t, s) f(s)$ for the Riemann-Stieltjes integral approximated by sums of the form $\sum_{i}\left[K\left(t, s_{i}\right)-K\left(t, s_{i-1}\right)\right] f\left(\xi_{i}\right)$, where $\left(\xi_{i}, s_{i}\right) \in T D_{[a, b]}$ is appropriate and $t \in[c, d]$ is given.

A proof of the next result can be found in [2] or in [16, Theorem II.1.1]. 
Theorem 2.3 (Bray). Suppose $\alpha \in S V([c, d], L(Y, Z)), f \in C([a, b], X)$ and $K \in C^{\sigma} \times(S V)^{u}([c, d] \times[a, b], L(X, Y))$ and define

$$
\begin{aligned}
& \left(F_{\alpha} K\right)(s)=\int_{[c, d]} d \alpha(t) \circ K(t, s), \quad s \in[a, b], \\
& \left(F_{K} f\right)(t)=\int_{[a, b]} d_{s} K(t, s) f(s), \quad t \in[c, d] .
\end{aligned}
$$

Then $F_{\alpha} K \in S V([a, b], L(X, Z))$ and $F_{K} f \in C([c, d], Y)$.

\subsection{The Fredholm Alternative and the Riemann-Stieltjes inte- grals.}

We now prove the conjecture of Hönig (see [17], the Remark after Theorem $\left.9^{c}\right)$.

Conjecture 2.1. Suppose $K \in C^{\sigma} \times\left(S V_{a}\right)^{u}([c, d] \times[a, b], L(X, Y))$ is such that given $t \in[c, d]$,

$$
K\left(t, s_{0}\right)^{*} y^{\prime}=\lim _{s \downarrow s_{0}} K(t, s)^{*} y^{\prime}
$$

for every $\left.s_{0} \in\right] a, b\left[\right.$ and every $y^{\prime} \in Y^{\prime}$. Let

$$
\left(F_{K}(f)\right)(t)=\int_{[a, b]} d_{s} K(t, s) f(s), \quad t \in[c, d],
$$

where $f \in C([a, b], X)$. Then $F_{K} \in L(C([a, b], X), C([c, d], Y))$ and the transposed operator $\left(F_{K}\right)^{*} \in L\left(B V_{c}^{+}\left([c, d], Y^{\prime}\right), B V_{a}^{+}\left([a, b], X^{\prime}\right)\right)$ is given by

$$
\left(\left(F_{K}\right)^{*}(y)\right)(s)=\int_{[c, d]} K(t, s)^{*} d y(t),
$$

for every $s \in[a, b]$ and every $y \in B V_{c}^{+}\left([c, d], Y^{\prime}\right)$.

Proof. It follows by straight application of Theorem 2.3 and the SaksHenstock Lemma (Lemma 2.1). Let $y \in B V_{c}^{+}\left([c, d], Y^{\prime}\right)$ and

$$
\left(\tilde{F}_{K^{*}}(y)\right)(s)=\int_{[c, d]} K(t, s)^{*} d y(t), \quad s \in[a, b] .
$$

Since

$$
\int_{[c, d]} K(t, s)^{*} d y(t)=\int_{[c, d]} d y(t) \circ K(t, s),
$$

then $\left(\tilde{F}_{K^{*}}(y)\right)(s)=\left(F_{y}(K)\right)(s)$ and Theorem 2.3 implies $\tilde{F}_{K^{*}} y \in S V([a, b]$, $L(X, \mathbb{R}))=B V\left([a, b], X^{\prime}\right)$. Therefore,

$$
\tilde{F}_{K^{*}} \in L\left(B V_{c}^{+}\left([c, d], Y^{\prime}\right), B V_{a}\left([a, b], X^{\prime}\right)\right) .
$$


It remains to prove that for every $y \in B V_{c}^{+}\left([c, d], Y^{\prime}\right)$ and every sufficiently small subinterval $\left[t_{0}, t_{0}+\rho\right], \rho>0$, of $[c, d]$,

$$
\lim _{s \downarrow s_{0}} \int_{\left[t_{0}, t_{0}+\rho\right]} K(t, s)^{*} d y(t)=\int_{\left[t_{0}, t_{0}+\rho\right]} K\left(t, s_{0}\right)^{*} d y(t),
$$

where $\left.s_{0} \in\right] a, b\left[\right.$ and $s>s_{0}$.

Let $\left.s_{0} \in\right] a, b[$ and $\left.s \in] s_{0}, b\right]$. Given $\varepsilon>0$, let $\delta$ be a constant gauge of $[c, d]$ such that for every $\delta$-fine $d=\left(\xi_{i}, t_{i}\right) \in T D_{[c, d]}$,

$$
\begin{aligned}
& \| \sum_{i=1}^{|d|} \int_{[c, d]}\left[K(t, s)^{*}-K\left(t, s_{0}\right)^{*}\right] d y(t) \\
& -\sum_{i=1}^{|d|}\left[K\left(\xi_{i}, s\right)^{*}-K\left(\xi_{i}, s_{0}\right)^{*}\right]\left[y\left(t_{i}\right)-y\left(t_{i-1}\right)\right] \|<\varepsilon,
\end{aligned}
$$

which means that $\left[K(\cdot, s)^{*}-K\left(\cdot, s_{0}\right)^{*}\right] \in R_{y}([c, d], L(X, Y))$. Let $t_{0} \in$ $[c, d]$ and $0<\rho<\delta / 2$. Then the pair $\left(t_{0},\left[t_{0}, t_{0}+\rho\right]\right)$ is a tagged partial division of $[c, d]$ which is $\delta$-fine and, therefore,

$$
\begin{aligned}
& \left\|\int_{\left[t_{0}, t_{0}+\rho\right]} K(t, s)^{*} d y(t)-\int_{\left[t_{0}, t_{0}+\rho\right]} K\left(t, s_{0}\right)^{*} d y(t)\right\| \\
& \leq \| \int_{\left[t_{0}, t_{0}+\rho\right]}\left[K(t, s)^{*}-K\left(t, s_{0}\right)^{*}\right] d y(t) \\
& -\left[K\left(t_{0}, s\right)^{*}-K\left(t_{0}, s_{0}\right)^{*}\right]\left[y\left(t_{0}+\rho\right)-y\left(t_{0}\right)\right] \| \\
& +\left\|\left[K\left(t_{0}, s\right)^{*}-K\left(t_{0}, s_{0}\right)^{*}\right]\left[y\left(t_{0}+\rho\right)-y\left(t_{0}\right)\right]\right\|,
\end{aligned}
$$

where the first summand is smaller than $\varepsilon$ by the Saks-Henstock Lemma (Lemma 2.1) and the second summand tends to zero as $s \rightarrow s_{0}$ by the left continuity of the function $K\left(t_{0}, \cdot\right)^{*}\left[y\left(t_{0}+\rho\right)-y\left(t_{0}\right)\right]$. The proof is complete.

We denote by $\mathcal{K}(X, Y)$ the subspace of $L(X, Y)$ of the compact linear operators and we write $\mathcal{K}(X)=\mathcal{K}(X, X)$. In accordance to the Remark after Theorem 15 in [17] and because Conjecture 2.1 is true, we have the following three theorems. For a proof of them consult [17].

Theorem 2.4. Suppose $K \in C^{\sigma} \times\left(S V_{a}\right)^{u}([a, b] \times[a, b], L(X))$. Given $t \in$ $[a, b]$, let

$$
K\left(t, s_{0}\right)^{*} x^{\prime}=\lim _{s \downarrow s_{0}} K(t, s)^{*} x^{\prime}
$$


for every $\left.s_{0} \in\right] a, b\left[\right.$ and every $x^{\prime} \in X^{\prime}$. Suppose the function

$$
K^{\square}: t \in[a, b] \mapsto K^{\square}(t)=K^{t} \in S V_{a}([a, b], L(X))
$$

belongs to $C\left([a, b], S V_{a}([a, b], \mathcal{K}(X))\right)$. Given $\lambda \in \mathbb{R}, \lambda \neq 0$, consider the integral equations

$$
\begin{gathered}
\lambda x(t)-\int_{[a, b]} d_{s} K(t, s) x(s)=f(t), \quad t \in[a, b], \\
\lambda u(t)-\int_{[a, b]} d_{s} K(t, s) u(s)=0, \quad t \in[a, b], \\
\lambda y(s)-\int_{[a, b]} K(t, s)^{*} d y(t)=g(s), \quad s \in[a, b], \\
\lambda z(s)-\int_{[a, b]} K(t, s)^{*} d z(t)=0, \quad s \in[a, b] .
\end{gathered}
$$

Then the Fredholm Alternative holds for these equations:

(a) either for every $f \in C([a, b], X)$, equation (4) has one and only one solution and the same applies to equation (6),

(b) or equation (5) has non-trivial solutions and the same applies to equation (7).

Theorem 2.5. Under the conditions of Theorem 2.4, if (b) holds, then equations (4) admits a solution if and only if $\int_{[a, b]} f(t) d z(t)=0$ for every solution $z$ of equation (7). Similarly, equation (6) admits a solution if and only if $\int_{[a, b]} u(t) d g(t)=0$ for every solution $u$ of equation (5).

Let $X$ be a Banach space. By $I_{X}$ or $I$ we mean the identical isomorphism of $X$.

Theorem 2.6. Under the conditions of Theorem 2.4, if (b) holds, then the dimension of the vector space of all solutions of equation (5) is finite and equals the dimension of the vector space of all solutions of equation (7) which is also equal to the codimension of $\left(\lambda I-F_{K}\right) C([a, b], X)$ in $C([a, b], X)$ and the codimension of $\left(\lambda I-\left(F_{K}\right)^{*}\right) B V_{b}^{+}\left([a, b], X^{\prime}\right)$ in $B V_{b}^{+}\left([a, b], X^{\prime}\right)$. 


\section{The integrals of Kurzweil and of Henstock}

\subsection{Definitions and terminology.}

Any function $f:[a, b] \rightarrow X$ is said to be Kurzweil integrable if there exists $I \in X$ (we write $I={ }^{K} \int_{[a, b]} f(t) d t$ ) such that given $\varepsilon>0$, there is a gauge $\delta$ of $[a, b]$ such that for every $\delta$-fine $d=\left(\xi_{i}, t_{i}\right) \in T D_{[a, b]}$,

$$
\left\|\sum_{i=1}^{|d|} f\left(\xi_{i}\right)\left(t_{i}-t_{i-1}\right)-I\right\|<\varepsilon .
$$

In this case, we write $f \in K([a, b], X)$. We use " $~ "$ to indicate the indefinite integral of a function $f \in K([a, b], X)$, that is,

$$
\tilde{f}(t)=K \int_{[a, t]} f(s) d s, \quad t \in[a, b] .
$$

If we consider only constant gauges in the definition of $f \in K([a, b], X)$, then we obtain the Riemann integral $\int_{[a, b]} f(t) d t$ and, when this is the case, we write $f \in R([a, b], X)$. Clearly $R([a, b], X) \subset K([a, b], X)$.

Any function $f:[a, b] \rightarrow X$ is said to be Henstock integrable (we write $f \in H([a, b], X))$ if there exists a function $F:[a, b] \rightarrow X$ such that given $\varepsilon>0$, there is a gauge $\delta$ of $[a, b]$ such that for every $\delta$-fine $d=\left(\xi_{i}, t_{i}\right) \in$ $T D_{[a, b]}$,

$$
\sum_{i=1}^{|d|}\left\|f\left(\xi_{i}\right)\left(t_{i}-t_{i-1}\right)-\left[F\left(t_{i}\right)-F\left(t_{i-1}\right)\right]\right\|<\varepsilon .
$$

In this case, we set ${ }^{H} \int_{[a, t]} f(s) d s=F(t)-F(a)$, for every $t \in[a, b]$.

It is immediate that the inclusion $H([a, b], X) \subset K([a, b], X)$ holds and if $f \in H([a, b], X)$, then $F(t)-F(a)=\tilde{f}(t)=K \int_{[a, t]} f(s) d s$, for every $t \in[a, b]$. When $X=\mathbb{R}$, then $H([a, b], X)=K([a, b], X)$ (see for instance $[25])$.

We use the notation ${ }^{K} \int$ and $\int$ to clearly distinguish between the integrals of Kurzweil and of Riemann (or Riemann-Stieltjes) respectively.

Let $f \in H([a, b], X)$ and $g:[a, b] \rightarrow X$ be such that $f=g$ almost everywhere in Lebesgue's sense. Then $g \in H([a, b], X)$ and $\widetilde{g}(t)=\tilde{f}(t)$, for every $t \in[a, b]$ (see for instance [6]). An analogous result holds for $K([a, b], X)$ instead of $H([a, b], X)$.

Two functions $f, g \in K([a, b], X)$ are said to be equivalent if and only if $\tilde{f}=\widetilde{g}$. We denote by $\mathbf{K}([\mathbf{a}, \mathbf{b}], \mathbf{X})$ (respectively by $\mathbf{H}([\mathbf{a}, \mathbf{b}], \mathbf{X})$ the space of all equivalence classes of functions of $K([a, b], X)$ (resp. of $H([a, b], X))$ 
equipped with the Alexiewicz norm

$$
\|f\|_{A}=\|\tilde{f}\|_{\infty}=\sup \left\{\left\|K \int_{[a, t]} f(s) d s\right\| ; t \in[a, b]\right\} .
$$

The spaces $\mathbf{K}([\mathbf{a}, \mathbf{b}], \mathbf{X})$ and $\mathbf{H}([\mathbf{a}, \mathbf{b}], \mathbf{X})$ are non-complete ([1], [18]), but they are ultrabornological ([11], Theorem 3.1 and Corollary 3.2).

As it should be expected, the integrals of Kurzweil and of Henstock are linear, additive over non-overlapping intervals and invariant over sets of Lebesgue measure zero. The indefinite integral of a Kurzweil (and hence of a Henstock) integrable function is continuous. See [12], [14], [22], [23], [25], [28]

For a proof of the next two results see [13] or [29].

Lemma 3.1 (Cousin Lemma). Given a gauge $\delta$ of $[a, b]$, there exists a $\delta$ fine $d=\left(\xi_{i}, t_{i}\right) \in T D_{[a, b]}$.

Lemma 3.2 (Saks-Henstock Lemma). If $\varepsilon>0$ and $\delta$ is a gauge of $[a, b]$ such that for every $\delta$-fine $d=\left(\xi_{i}, t_{i}\right) \in T D_{[a, b]}$,

$$
\left\|K \int_{[a, b]} f(t) d t-\sum_{i=1}^{|d|} f\left(\xi_{i}\right)\left(t_{i}-t_{i-1}\right)\right\|<\varepsilon,
$$

then for every $\delta$-fine $d=\left(\zeta_{j}, s_{j}\right) \in T P D_{[a, b]}$,

$$
\left\|\sum_{j=1}^{|d|}\left\{K \int_{\left[s_{j-1}, s_{j}\right]} f(t) d t-f\left(\zeta_{j}\right)\left(s_{j}-s_{j-1}\right)\right\}\right\|<\varepsilon .
$$

The result that follows is due to Hönig and can be found in [18] or in [7, Theorem 6].

Theorem 3.1. Consider $\alpha \in S V([a, b], L(X, Y))$ and $f \in K([a, b], X)$. Then $\alpha(\cdot) f(\cdot) \in K([a, b], Y)$ with

$$
\begin{aligned}
K \int_{[a, b]} \alpha(t) f(t) d t & =\int_{[a, b]} \alpha(t) d \tilde{f}(t) \quad \text { and } \\
\left\|K \int_{[a, b]} \alpha(t) f(t) d t\right\| & \leq[V(\alpha)+\|\alpha(a)\|]\|f\|_{A} .
\end{aligned}
$$

In an analogous way, it can be proved that 
Theorem 3.2. If $\alpha \in K([a, b], L(X, Y))$ and $f \in B V([a, b], X)$, then we have $\alpha(\cdot) f(\cdot) \in K([a, b], Y)$ with

$$
\begin{aligned}
K \int_{[a, b]} \alpha(t) f(t) d t & =\int_{[a, b]} d \tilde{\alpha}(t) f(t) \quad \text { and } \\
\left\|K \int_{[a, b]} \alpha(t) f(t) d t\right\| & \leq\|\alpha\|_{A}[V(f)+\|f(a)\|] .
\end{aligned}
$$

A proof of Theorem 3.2 can be found in [7, Theorem 9].

Any function $F:[a, b] \rightarrow X$, is said to be differentiable at $\xi \in] a, b[$ with derivative $D F(t)$, whenever for every $\varepsilon>0$, there is an open neighborhood $V \subset[a, b]$ of $t$ such that

$$
\|F(v)-F(u)-f(t)(v-u)\|<\varepsilon(v-u)
$$

for every subinterval $[u, v] \subset[a, b]$ with $t \in[u, v] \subset V$. Then $F$ is differentiable almost everywhere (in Lebesgue's sense) if $F$ is differentiable at $t$ for almost every $t \in] a, b[$.

We say that a function $\alpha:[a, b] \rightarrow L(X, Y)$ is weakly differentiable almost everywhere if for every $x \in X$, the function

$$
\alpha(\cdot) x: t \in] a, b[\mapsto \alpha(t) x \in Y
$$

is differentiable almost everywhere. We denote by $D^{\sigma}(\alpha x)(t)$ the derivative of $\alpha(\cdot) x$ at $t$.

For a proof of the next two theorems, see [6].

Theorem 3.3. If $f \in H([a, b], X)$, then its indefinite integral is differentiable almost everywhere and $D \tilde{f}(t)=f(t)$ for almost every $t \in[a, b]$.

Theorem 3.4. If $\alpha:[a, b] \rightarrow L(X, Y)$ is differentiable almost everywhere and $f \in H([a, b], X)$, then the function $g_{\alpha, f}(t)=D^{\sigma}(\alpha f(t))(t)$ belongs to $H([a, b], Y)$ and

$$
K \int_{[a, b]} g_{\alpha, f}(t) d t=K \int_{[a, b]} d \alpha(t) f(t) .
$$

\subsection{Auxiliary results.}

Suppose $\alpha \in S V([a, b], L(X, Y))$ and $f \in C([a, b], X)$. Then the Riemann-Stieltjes integral $\int_{[a, b]} d \alpha(t) f(t)$ exists (Theorem 2.1). Furthermore, if we define

$$
F_{\alpha}(f)=\int_{[a, b]} d \alpha(t) f(t),
$$

then $F_{\alpha} \in L(C([a, b], X), Y)$ and $\left\|F_{\alpha}\right\| \leq S V(\alpha)$ (see [16, Theorem I.4.6]). 
If $\alpha \in S V([a, b], L(X, Y))$, then for every $t \in[a, b[$ there is one and only one element $\alpha(t \dot{+}) \in L\left(X, Y^{\prime \prime}\right)$ such that for every $x \in X$ and every $y^{\prime} \in Y^{\prime}$

$$
\lim _{\rho \downarrow 0}\left\langle\alpha(t+\rho) x, y^{\prime}\right\rangle=\left\langle\alpha(t \dot{+}) x, y^{\prime}\right\rangle
$$

where $\rho>0$ ([15, Corollary after I.3.6]). Thus given $\alpha \in S V([a, b], L(X, Y))$, we define $\alpha^{+}(t)=\alpha(t \dot{+}), a<t<b$, and $\alpha^{+}(a)=\alpha(a)$. Then the function $\alpha^{+}$is of bounded semi-variation and, when $\alpha(a \dot{+})=\alpha(a)$, we write $\alpha^{+} \in S V^{+}\left([a, b], L\left(X, Y^{\prime \prime}\right)\right)$. If in addition $\alpha^{+}(b)=0$, then we write $\alpha^{+} \in S V_{b}^{+}\left([a, b], L\left(X, Y^{\prime \prime}\right)\right)$. Moreover for every $f \in C([a, b], X)$,

$$
\int_{[a, b]} d \alpha^{+}(t) f(t)=\int_{[a, b]} d \alpha(t) f(t)
$$

and $\left\|F_{\alpha}\right\|=S V\left(\alpha^{+}\right)([15$, Corollary 3.9] $)$.

Given a function $\alpha:(t, s) \in[c, d] \times[a, b] \mapsto \alpha(t, s) \in L\left(X, Y^{\prime \prime}\right)$, we write $\alpha^{t}(s)=\alpha_{s}(t)=\alpha(t, s)$. Suppose that

- given $s \in[a, b]$, the function

$$
h_{\alpha, x, s}: t \in[c, d] \mapsto h_{\alpha, x, s}(t)=\int_{[a, s]} \alpha(t, \rho) x d \rho
$$

is continuous for every $x \in X$, and

- given $t \in[c, d], \alpha^{t} \in S V_{b}^{+}\left([a, b], L\left(X, Y^{\prime \prime}\right)\right)$ and

$$
S V^{u}(\alpha)=\sup \left\{S V\left(\alpha^{t}\right) ; t \in[a, b]\right\}<\infty .
$$

In this case, we write $\alpha \in \tilde{C}^{\sigma} \times\left(S V_{b}^{+}\right)^{u}\left([c, d] \times[a, b], L\left(X, Y^{\prime \prime}\right)\right)$. When we consider functions of bounded variation instead of functions of bounded semi-variation, we write $\alpha \in \tilde{C}^{\sigma} \times\left(B V_{b}^{+}\right)^{u}\left([c, d] \times[a, b], L\left(X, Y^{\prime \prime}\right)\right)$.

Suppose now that $\alpha \in \tilde{C}^{\sigma} \times\left(B V_{b}^{+}\right)^{u}\left([c, d] \times[a, b], L\left(X, Y^{\prime \prime}\right)\right)$ with $\int_{[c, t]} \alpha(t, \rho) x d \rho \in Y$ for all $t \in[c, d]$ and $x \in X$. Under these circumstances we have

Theorem 3.5. If $K:[c, d] \times[a, b] \rightarrow L(X, Y)$ is such that for every $x \in X$,

$$
K(t, s) x=\int_{[c, t]} \alpha(t, \rho) x d \rho
$$

then $K \in C^{\sigma} \times\left(S V_{a}\right)^{u}([c, d] \times[a, b], L(X, Y))$ and for every $t \in[c, d]$, every $\left.s_{0} \in\right] a, b\left[\right.$ and every $y^{\prime} \in Y^{\prime}$,

$$
K\left(t, s_{0}\right)^{*} y^{\prime}=\lim _{s \rightarrow s_{0}} K(t, s)^{*} y^{\prime} .
$$


Proof. By the hypothesis with respect to the first variable of $\alpha=\alpha(t, s)$, for every $s \in[a, b]$ and every $x \in X$,

$$
h_{\alpha, x, s}: t \in[c, d] \mapsto h_{\alpha, x, s}(t)=\int_{[a, s]} \alpha(t, \rho) x d \rho
$$

is a continuous function. Hence $K_{s}:[c, d] \rightarrow L(X, Y)$ is weakly continuous for every $s \in[a, b]$. Let $d=\left(s_{i}\right) \in D_{[a, b]}$. Then for every $t \in[c, d]$ and every $x_{i} \in X$ with $\left\|x_{i}\right\| \leq 1, i=1,2, \ldots,|d|$,

$$
\begin{aligned}
& \left\|\sum_{i=1}^{|d|}\left[K\left(t, s_{i}\right)-K\left(t, s_{i-1}\right)\right] x_{i}\right\|=\left\|\sum_{i=1}^{|d|} \int_{\left[s_{i-1}, s_{i}\right]} \alpha(t, \rho) x_{i} d \rho\right\| \\
& \leq \sum_{i=1}^{|d|}\left\|\alpha^{t}\right\|_{\infty}\left\|x_{i}\right\|\left(s_{i}-s_{i-1}\right) \leq\left\|\alpha^{t}\right\|_{\infty}(b-a) \leq V\left(\alpha^{t}\right)(b-a)
\end{aligned}
$$

which implies $K^{t} \in S V_{a}([a, b], L(X, Y))$. Hence $K \in C^{\sigma} \times\left(S V_{a}\right)^{u}([c, d] \times$ $[a, b], L(X, Y))$. By the continuity of the indefinite integral, the function $K^{t}(\cdot) x: s \in[a, b] \mapsto K(t, s) x=\int_{[c, t]} \alpha(t, \rho) x d \rho$ is continuous for every $t \in[c, d]$ and every $x \in X$. Hence, given $t \in[c, d]$ and $y^{\prime} \in Y^{\prime},\left(K^{t}(\cdot)\right)^{*} y^{\prime}$ is also a continuous function on $[a, b]$.

Theorem 3.6. Under the conditions of Theorem 3.5,

$$
\int_{[a, b]} d_{s} K(t, s) y(s)=\int_{[a, b]} \alpha(t, s) y(s) d s
$$

for every $y \in C([a, b], X)$ and every $t \in[c, d]$.

Proof. Let $y \in C([a, b], X), t \in[c, d]$ and $g=\tilde{y}$, that is, $g(s)=\int_{[a, s]} y(r) d r$ for every $s \in[a, b]$. By Theorem 2.2, $\alpha^{t}(\cdot) y(\cdot)$ is Riemann integrable and

$$
\int_{[a, b]} \alpha^{t}(s) y(s) d s=\int_{[a, b]} \alpha^{t}(s) d g(s)=-\int_{[a, b]} d_{s}\left(\alpha^{t}(s)\right) \beta(s),
$$

where we applied Theorem 2.1 to obtain the last equality. Also by Theorem 2.1, the Riemann-Stieltjes integral $\int_{[a, b]} d_{s} K(t, s) y(s)$ exists for every $t \in$ $[c, d]$, since $K^{t} \in S V([a, b], L(X, Y))$ and $y \in C([a, b], X)$.

Because $\alpha^{t}(\cdot) x \in H([a, b] Y)$ (Theorem 3.1) and $K(t, s) x=\int_{[a, s]} \alpha^{t}(\rho) x d \rho$ for all $x \in X, t \in[c, d]$ and $s \in[a, b]$, the Fundamental Theorem of Calculus (Theorem 3.3) implies that given $t \in[c, d]$ and $x \in X$, the derivative (with respect to $s$ ) of the indefinite integral of $\alpha^{t}(\cdot) x$ exists and

$$
D_{s}^{\sigma}(K(t, s) x)=D_{s}^{\sigma} \int_{[a, s]} \alpha^{t}(\rho) x d \rho=\alpha^{t}(s) x
$$


for almost every $s \in[a, b]$. But by Theorem 3.4,

$$
\int_{[a, b]} D_{s}^{\sigma}(K(t, s) x) d s=\int_{[a, b]} d_{s} K(t, s) x .
$$

Hence,

$$
\int_{[a, b]} d_{s} K(t, s) x=\int_{[a, b]} \alpha^{t}(s) x d s,
$$

for every $x \in X$ and every $t \in[c, d]$. It follows then that

$$
\int_{[a, b]} d_{s} K(t, s) g(s)=\int_{[a, b]} \alpha(t, s) g(s) d s,
$$

for every step function $g:[a, b] \rightarrow X$.

Let $\varepsilon>0$. Consider $g:[a, b] \rightarrow X$ a step function such that $\|g-y\|_{\infty}<$ $\varepsilon / N$, where $N=\max \left\{S V\left(K^{t}\right), V\left(\alpha^{t}\right)\right\}, t \in[c, d]$ fixed. Let $\delta_{1}$ and $\delta_{2}$ be constant gauges of $[a, b]$ from the definitions of the integrals

$$
\int_{[a, b]} d_{s} K(t, s)[y(s)-g(s)] \text { and } \int_{[a, b]} \alpha(t, s)[y(s)-g(s)] d s
$$

respectively. Define $\delta=\min \left\{\delta_{i} ; i=1,2\right\}$ and let $d=\left(\xi_{i}, s_{i}\right) \in T D_{[a, b]}$ be $\delta$-fine. Hence,

$$
\begin{aligned}
& \left\|\int_{[a, b]} d_{s} K(t, s) y(s)-\int_{[a, b]} \alpha(t, s) y(s) d s\right\| \\
& \leq\left\|\int_{[a, b]} d_{s} K(t, s)[y(s)-g(s)]\right\| \\
& +\left\|\int_{[a, b]} d_{s} K(t, s) g(s)-\int_{[a, b]} \alpha(t, s) g(s) d s\right\| \\
& +\left\|\int_{[a, b]} \alpha(t, s)[y(s)-g(s)] d s\right\|,
\end{aligned}
$$

where the second summand is equal to zero by (10), the first summand is smaller than

$$
\begin{aligned}
& \left\|\int_{[a, b]} d_{s} K(t, s)[y(s)-g(s)]-\sum_{i=1}^{|d|}\left[K\left(t, s_{i}\right)-K\left(t, s_{i-1}\right)\right]\left[y\left(\xi_{i}\right)-g\left(\xi_{i}\right)\right]\right\| \\
& +\left\|\sum_{i=1}^{|d|}\left[K\left(t, s_{i}\right)-K\left(t, s_{i-1}\right)\right]\left[y\left(\xi_{i}\right)-g\left(\xi_{i}\right)\right]\right\| \\
& <\varepsilon+S V\left(K^{t}\right)\|y-g\|_{\infty}<2 \varepsilon,
\end{aligned}
$$


and the third summand is smaller than $V\left(\alpha^{t}\right)\|y-g\|_{\infty}<\varepsilon$. The result follows.

Let $X$ be a Banach space. By $G([a, b], X)$ we mean the space of all regulated functions $f:[a, b] \rightarrow X$, that is, there exist

$$
f(t+)=\lim _{\rho \downarrow 0} f(t+\rho), \text { for every } t \in[a, b[,
$$

and

$$
\left.\left.f(t-)=\lim _{\rho \downarrow 0} f(t-\rho), \text { for every } t \in\right] a, b\right],
$$

where $\rho>0$. When equipped with the usual supremum norm, $G([a, b], X)$ is complete ([15], [16]).

Given a function $f:[a, b] \rightarrow X$ and a division $d=\left(t_{i}\right) \in D_{[a, b]}$, we define

$$
\omega_{i}^{\circ}(f)=\sup \{\|f(t)-f(s)\| ; t, s \in] t_{i-1} t_{i}[\},
$$

for each $i=1,2, \ldots,|d|$, and

$$
\omega_{d}^{\circ}(f)=\sup _{i}\left\{\omega_{i}^{\circ}(f)\right\} .
$$

Then a subset $E \subset G([a, b], X)$ is called equiregulated if given $\varepsilon>0$, there exists $d=\left(t_{i}\right) \in D_{[a, b]}$ such that for every function $f \in E, \omega_{d}^{\circ}(f)<\varepsilon$.

Remark 3.1. It is not difficult to verify that

(i) $C([a, b], X) \subset G([a, b], X)$;

(ii) $E \subset C([a, b], X)$ is equiregulated if and only if $E$ is equicontinuous.

We call any subset $A \subset X$ relatively compact if the closure of $A$ in $X$ is compact.

Lemma 3.3. Suppose $\alpha \in S V([a, b], L(X, Y)), f \in C([a, b], X)$ and

$$
F_{\alpha}(f)=\int_{[a, b]} d \alpha(t) f(t) .
$$

Then $F_{\alpha}$ maps bounded and equiregulated subsets of $C([a, b], X)$ to relatively compact subsets of $Y$ if and only if $\alpha(t) \in \mathcal{K}(X, Y)$, for every $t \in[a, b]$.

For a proof of Lemma 3.3, see [17, Theorem 4d]. As a direct consequence of Lemma 3.3 we have

Lemma 3.4. Suppose $K \in C^{\sigma} \times\left(S V_{a}\right)^{u}([c, d] \times[a, b], L(X, Y)), f \in C([a, b]$, $X)$ and

$$
\left(F_{K} f\right)(t)=\int_{[a, b]} d_{s} K(t, s) f(s), \quad t \in[c, d]
$$


If for every bounded and equiregulated subset $E$ of $C([a, b], X)$, the subset $\left\{\left(F_{K} f\right)(t) ; f \in E\right\}$ of $Y$ is relatively compact for every $t \in[c, d]$, then $K(t, s) \in \mathcal{K}(X, Y)$ for every $(t, s) \in[c, d] \times[a, b]$.

Theorem 3.7. Suppose the hypotheses of Theorem 3.5 are fulfilled. If for every $t \in[c, d]$ and every $s \in[a, b], \alpha^{t}(s) \in \mathcal{K}(X, Y)$, then the function

$$
K^{\square}: t \in[a, b] \mapsto K^{\square}(t)=K^{t} \in S V_{a}([a, b], L(X, Y))
$$

belongs to $C\left([a, b], S V_{a}([a, b], \mathcal{K}(X, Y))\right)$.

Proof. At first we will show that $K^{\square} \in C\left([a, b], S V_{a}([a, b], L(X, Y))\right)$. Let $t_{0} \in[c, d]$ and $d=\left(s_{i}\right) \in D_{[a, b]}$. By the hypothesis for $h_{\alpha, x, s}(t)=$ $\int_{[a, s]} \alpha(t, \rho) x d \rho$, given $\varepsilon>0$, there exists $\delta_{i}>0$ such that $0<\left|t-t_{0}\right|<\delta_{i}$ implies

$$
\left\|\int_{\left[s_{i-1}, s_{i}\right]}\left[\alpha(t, \rho)-\alpha\left(t_{0}, \rho\right)\right] x_{i} d \rho\right\|<\frac{\varepsilon}{2^{i}},
$$

where $x_{i} \in X, i=1,2, \ldots,|d|$. Then, for $\left\|x_{i}\right\| \leq 1, i=1,2, \ldots,|d|$,

$$
\begin{aligned}
& \left\|\sum_{i=1}^{|d|}\left[\left(K^{t}-K^{t_{0}}\right)\left(s_{i}\right)-\left(K^{t}-K^{t_{0}}\right)\left(s_{i-1}\right)\right] x_{i}\right\| \\
& \leq \sum_{i=1}^{|d|}\left\|\int_{\left[s_{i-1}, s_{i}\right]}\left[\alpha(t, \rho)-\alpha\left(t_{0}, \rho\right)\right] x_{i} d \rho\right\|=\varepsilon .
\end{aligned}
$$

Therefore

$$
\begin{aligned}
& \left\|K^{t}-K^{t_{0}}\right\|=S V\left(K^{t}-K^{t_{0}}\right) \\
& =\sup _{d} \sup \left\{\left\|\sum_{i=1}^{|d|}\left[\left(K^{t}-K^{t_{0}}\right)\left(s_{i}\right)-\left(K^{t}-K^{t_{0}}\right)\left(s_{i-1}\right)\right] x_{i}\right\| ; x_{i} \in Y,\left\|x_{i}\right\| \leq 1\right\}
\end{aligned}
$$

which tends to zero as $t \rightarrow t_{0}$.

It remains to prove that $K(t, s) \in \mathcal{K}(X, Y)$ for every $(t, s) \in[c, d] \times[a, b]$. Given $y \in C([a, b], X)$, define

$$
\left(F_{K}(y)\right)(t)=\int_{[a, b]} d_{s} K(t, s) y(s), \quad t \in[c, d],
$$

and

$$
F_{\alpha^{t}}(y)=\int_{[a, b]} d_{s}\left(\alpha^{t}(s)\right) \tilde{y}(s) .
$$

Let $E \subset C([a, b], X)$ be equiregulated and bounded and let $M>0$ be such that $\|y\|_{\infty}<M$ for every $y \in E$. Let $A=\{\tilde{y} ; y \in E\}$, that is, $A$ is the subset of all indefinite integrals of elements of $E$. We will prove that $A$ is 
equiregulated. By Remark 3.1, it is enough to show that $A$ is equicontinuous which is true: let $s \in[a, b], \varepsilon>0$ and $\delta=\varepsilon / M$, then for every $\tilde{y} \in A$ and every $r \in[s, b]$ with $0<r-s<\delta$,

$$
\|\tilde{y}(s)-\tilde{y}(r)\| \leq \int_{[s, r]}\|y(\rho)\| d \rho<M(r-s)<\varepsilon
$$

and a similar inequality holds for $r \in[a, s]$ with $0<s-r<\delta$. Now, according to equations (9) and (8) (see Theorem 3.6 and its proof),

$$
\int_{[a, b]} d_{s} K(t, s) y(s)=-\int_{[a, b]} d_{s}\left(\alpha^{t}(s)\right) \tilde{y}(s)
$$

and then

$$
\left(F_{K}(E)\right)(t)=\left\{\left(F_{K}(y)\right)(t) ; y \in E\right\}=\left\{-F_{\alpha^{t}}(\tilde{y}) ; y \in E\right\},
$$

for every $t \in[c, d]$. Thus, $\left(F_{K}(E)\right)(t)=-F_{\alpha^{t}}(A)$ is relatively compact by Lemma 3.3. Finally, by Lemma 3.4, $K(t, s) \in \mathcal{K}(X, Y)$ for every $(t, s) \in$ $[c, d] \times[a, b]$ and the proof is finished.

\subsection{The Fredholm Alternative and the Kurzweil integral.}

As defined earlier, $\mathbf{K}([\mathbf{a}, \mathbf{b}], \mathbf{X})$ is the space of all equivalence classes of functions of $K([a, b], X)$ endowed with the Alexiewicz norm. The result that follows is due to Hönig. For a proof of it consult [19, Theorem 2.6] or, alternatively, [10, Theorem 3.5]).

Suppose $\alpha \in \tilde{C}^{\sigma} \times\left(S V_{b}^{+}\right)^{u}\left([c, d] \times[a, b], L\left(X, Y^{\prime \prime}\right)\right)$ with $\int_{[c, t]} \alpha(t, \rho) x d \rho \in$ $Y$ and

$$
\left(H_{\alpha}(f)\right)(t)=K \int_{[a, b]} \alpha(t, s) f(s) d s
$$

for all $t \in[c, d]$ and $x \in X$. Under these conditions we have

Theorem 3.8. The mapping

$$
\alpha \mapsto H_{\alpha} \in L(\mathbf{K}([\mathbf{a}, \mathbf{b}], \mathbf{X}), \mathbf{C}([\mathbf{c}, \mathbf{d}], \mathbf{Y})),
$$

is an isometry (i.e., $\left\|H_{\alpha}\right\|=S V^{u}(\alpha)$ ) onto. Furthermore for every $t \in$ $[c, d]$, every $s \in[a, b]$ and every $x \in X$,

$$
\int_{[a, s]} \alpha(t, \rho) x d \rho=\left(H_{\alpha}\left(\chi_{[a, s]} x\right)\right)(t) .
$$

The next theorem is Hönig's result for the linear integral equation concerning the integral of Kurzweil mentioned as result (B) in the introduction. It can be found in [19, Theorem 3.2]. 
Theorem 3.9. Given $H \in L(\mathbf{K}([\mathbf{a}, \mathbf{b}], \mathbf{X}), \mathbf{C}([\mathbf{a}, \mathbf{b}], \mathbf{X}))$, let $\alpha:[a, b] \times$ $[a, b] \rightarrow L\left(X, X^{\prime \prime}\right)$ be the corresponding kernel by Theorem 3.8 (i.e., $H=$ $\left.H_{\alpha}\right)$. Suppose $H$ is such that for every $f \in \mathbf{K}([\mathbf{a}, \mathbf{b}], \mathbf{X})$, the linear FredholmKurzweil integral equation

$$
x(t)-K \int_{[a, b]} \alpha(t, s) x(s) d s=f(t), \quad t \in[a, b] .
$$

(i.e., the equation $x-H(x)=f$ ) admits a unique solution $x_{f} \in \mathbf{K}([\mathbf{a}, \mathbf{b}], \mathbf{X})$. Then there is a unique kernel $\phi \in \tilde{C}^{\sigma} \times\left(S V_{b}^{+}\right)^{u}\left([a, b] \times[a, b], L\left(X, X^{\prime \prime}\right)\right)$ with $\int_{[a, t]} \phi(t, \rho) x d \rho \in X$ for every $t \in[a, b]$ and every $x \in X$, and such that given $f \in \mathbf{K}([\mathbf{a}, \mathbf{b}], \mathbf{X})$,

$$
x_{f}(t)=f(t)-K \int_{[a, b]} \phi(t, s) f(s) d s, \quad t \in[a, b] .
$$

The following observations are borrowed from [19].

Remark 3.2. Suppose $E$ is a dense subspace of $\mathbf{K}([\mathbf{a}, \mathbf{b}], \mathbf{X})$ that contains $C([a, b], X)$ and $H \in L(E, C([a, b], X))$ is such that for every $f \in E$, the equation $x-H(x)=f$ has one and only one solution $x_{f} \in E$, then Theorem 3.9 still holds if we replace $\mathbf{K}([\mathbf{a}, \mathbf{b}], \mathbf{X})$ by $E$. As an example we can mention $R^{2}([a, b], X)$, the space of all functions from $[a, b]$ to $X$ which are improper Riemann integrable with a finite number of points of improper integration. This space is also ultrabornological ([11, Corollary 3.2]).

Now we are able to present the main result on the Fredholm Alternative for equation (11). Assume $\alpha \in \tilde{C}^{\sigma} \times\left(B V_{b}^{+}\right)^{u}\left([a, b] \times[a, b], L\left(X, X^{\prime \prime}\right)\right)$ is such that for every $t \in[a, b]$, every $s \in[a, b]$ and every $x \in X, \alpha^{t}(s) \in \mathcal{K}(X)$ and $\int_{[a, t]} \alpha(t, \rho) x d \rho \in X$. Under these conditions we have

Theorem 3.10. Suppose $f \in \mathbf{K}([\mathbf{a}, \mathbf{b}], \mathbf{X})$ and consider the linear FredholmKurzweil integral equation

$$
x(t)-K \int_{[a, b]} \alpha(t, s) x(s) d s=f(t), \quad t \in[a, b],
$$

and its corresponding homogeneous equation

$$
u(t)-K \int_{[a, b]} \alpha(t, s) u(s) d s=0, \quad t \in[a, b] .
$$

Consider also the integral equations

$$
y(s)-\int_{[a, s]}\left[\int_{[a, b]} \alpha(t, s)^{*} d y(t)\right] d s=g(s), \quad s \in[a, b],
$$


and

$$
z(s)-\int_{[a, s]}\left[\int_{[a, b]} \alpha(t, s)^{*} d z(t)\right] d s=0, \quad s \in[a, b],
$$

where $y, z \in C\left([a, b], X^{\prime}\right)$. Then

(a) either for every $f \in \mathbf{K}([\mathbf{a}, \mathbf{b}], \mathbf{X})$, equation (12) has a unique solution $x_{f} \in \mathbf{K}([\mathbf{a}, \mathbf{b}], \mathbf{X})$ and, for every $g \in C\left([a, b], X^{\prime}\right)$, equation (14) has a unique solution $y_{g} \in C\left([a, b], X^{\prime}\right)$;

(b) or equations (13) and (15) admit non-trivial solutions respectively in $\mathbf{K}([\mathbf{a}, \mathbf{b}], \mathbf{X})$ and in $C\left([a, b], X^{\prime}\right)$. In this case, equation (12) admits a solution if and only if for every solution $z \in C\left([a, b], X^{\prime}\right)$ of equation (15) we have

$$
\int_{[a, b]}\left[K \int_{[a, b]} \alpha(t, s) f(s) d s\right] d z(t)=0 .
$$

Proof. Let $v=x-f$ and $h(t)={ }^{K} \int_{[a, b]} \alpha(t, s) f(s) d s, t \in[a, b]$. Then

$$
v(t)=K \int_{[a, b]} \alpha(t, s) x(s) d s, \quad t \in[a, b] .
$$

By Theorem 3.8, the functions $h$ and $v$ belong to $C([a, b], X)$, because for every $f, x \in \mathbf{K}([\mathbf{a}, \mathbf{b}], \mathbf{X})$ and every $t \in[a, b]$, we have $h(t)=\left(H_{\alpha}(f)\right)(t)$, $v(t)=\left(H_{\alpha}(x)\right)(t)$, and $H_{\alpha} \in L(\mathbf{K}([\mathbf{a}, \mathbf{b}], \mathbf{X}), \mathbf{C}([\mathbf{a}, \mathbf{b}], \mathbf{X}))$. Thus equation (12) is equivalent to the integral equation

$$
v(t)-K \int_{[a, b]} \alpha(t, s) v(s) d s=h(t), \quad t \in[a, b] .
$$

But according to Theorem 2.2, equation (17) is in fact

$$
v(t)-\int_{[a, b]} \alpha(t, s) v(s) d s=h(t), \quad t \in[a, b],
$$

where the integral is that of Riemann. Let $K:[a, b] \times[a, b] \rightarrow L(X)$ be such that for every $(t, s) \in[a, b] \times[a, b]$ and every $x \in X, K(t, s) x=$ $\int_{[a, s]} \alpha(t, \rho) x d \rho$. By Theorem 3.5,

$$
\int_{[a, b]} d_{s} K(t, s) v(s)=\int_{[a, b]} \alpha(t, s) v(s) d s
$$

and therefore equation (18) is equivalent to the next Riemann-Stieltjes integral equation

$$
v(t)-\int_{[a, b]} d_{s} K(t, s) v(s)=h(t), \quad t \in[a, b] .
$$


Also by Theorem 3.5, $K \in C^{\sigma} \times\left(S V_{a}\right)^{u}([a, b] \times[a, b], L(X))$ and, for every $t \in[a, b]$, every $\left.s_{0} \in\right] a, b\left[\right.$ and every $x^{\prime} \in X^{\prime}$,

$$
K\left(t, s_{0}\right)^{*} x^{\prime}=\lim _{s \rightarrow s_{0}} K(t, s)^{*} x^{\prime} .
$$

Moreover, $K^{\square} \in C\left([a, b], S V_{a}([a, b], \mathcal{K}(X))\right)$ where $K^{\square}(t)=K^{t}$ (see Theorem 3.7). Hence by Theorems 2.4 and 2.5 it follows that

$\left(\mathrm{a}_{0}\right)$ either for every $h \in C_{a}([a, b], X)$, equation (19) has one and only one solution $v_{h} \in C_{a}([a, b], X)$ and, for every $g \in C_{a}\left([a, b], X^{\prime}\right)$, the equation

$$
y(s)-\int_{[a, b]} K(t, s)^{*} d y(t)=g(s), \quad s \in[a, b],
$$

has one and only one solution $y_{g} \in C_{a}\left([a, b], X^{\prime}\right)$;

$\left(b_{0}\right)$ or equations

$$
r(t)-\int_{[a, b]} d_{s} K(t, s) r(s)=0, \quad t \in[a, b],
$$

and

$$
z(s)-\int_{[a, b]} K(t, s)^{*} d z(t)=0, \quad s \in[a, b],
$$

admit non-trivial solutions. In this case, equation (19) has a solution if and only if

$$
\int_{[a, b]} h(t) d z(t)=0
$$

for every solution $z \in C_{a}\left([a, b], X^{\prime}\right)$ of $(22)$.

Now, by the definition of $K$ it follows that $K(t, s)^{*} x^{\prime}=\int_{[a, b]} \alpha(t, \rho)^{*} x^{\prime} d \rho$, for every $(t, s) \in[a, b] \times[a, b]$ and every $x^{\prime} \in X^{\prime}$, by taking approximated Riemann sums. Also by approximated Riemann sums we prove that

$$
\int_{[a, b]} K(t, s)^{*} d y(t)=\int_{[a, s]}\left[\int_{[a, b]} \alpha(t, s)^{*} d y(t)\right] d s, \quad s \in[a, b],
$$

for every $y \in C\left([a, b], X^{\prime}\right)$. Let $s \in[a, b]$. Given $\varepsilon>0$, let $\delta$ be a constant gauge of $[a, b]$ such that for every $d=\left(\xi_{i}, t_{i}\right) \in T D_{[a, b]}$,

$$
\left\|\int_{[a, b]} K(t, s)^{*} d y(t)-\sum_{i=1}^{|d|} K\left(\xi_{i}, s\right)^{*}\left[y\left(t_{i}\right)-y\left(t_{i-1}\right)\right]\right\|<\varepsilon
$$

and

$$
\left\|\int_{[a, b]} \alpha(t, s)^{*} d y(t)-\sum_{i=1}^{|d|} \alpha\left(\xi_{i}, s\right)^{*}\left[y\left(t_{i}\right)-y\left(t_{i-1}\right)\right]\right\|<\varepsilon .
$$


Then

$$
\begin{aligned}
& \left\|\int_{[a, b]} K(t, s)^{*} d y(t)-\int_{[a, s]}\left[\int_{[a, b]} \alpha(t, s)^{*} d y(t)\right] d s\right\| \\
& \leq\left\|\int_{[a, b]} K(t, s)^{*} d y(t)-\sum_{i=1}^{|d|} K\left(\xi_{i}, s\right)^{*}\left[y\left(t_{i}\right)-y\left(t_{i-1}\right)\right]\right\| \\
& +\left\|\sum_{i=1}^{|d|} K\left(\xi_{i}, s\right)^{*}\left[y\left(t_{i}\right)-y\left(t_{i-1}\right)\right]-\int_{[a, s]}\left[\sum_{i=1}^{|d|} \alpha\left(\xi_{i}, s\right)^{*}\left[y\left(t_{i}\right)-y\left(t_{i-1}\right)\right]\right] d s\right\| \\
& +\left\|\int_{[a, s]}\left[\sum_{i=1}^{|d|} \alpha\left(\xi_{i}, s\right)^{*}\left[y\left(t_{i}\right)-y\left(t_{i-1}\right)\right]\right] d s-\int_{[a, s]}\left[\int_{[a, b]} \alpha(t, s)^{*} d y(t)\right] d s\right\|
\end{aligned}
$$

where the first summand is smaller than $\varepsilon$, the third summand is smaller than $\varepsilon(s-a)$, and the second summand is equal to

$$
\left\|\sum_{i=1}^{|d|} K\left(\xi_{i}, s\right)^{*}\left[y\left(t_{i}\right)-y\left(t_{i-1}\right)\right]-\sum_{i=1}^{|d|} \int_{[a, s]} \alpha\left(\xi_{i}, s\right)^{*}\left[y\left(t_{i}\right)-y\left(t_{i-1}\right)\right] d s\right\|=0 .
$$

Therefore equations (20) and (14) are equivalent and the same applies to equations (22) and (15). By (23) and the definition of $h$, we have (16) and the result follows.

The next result is a consequence of Theorem 3.9.

Theorem 3.11. Under the conditions required for Theorem 3.10, if (a) holds, then there is a unique kernel $\phi \in \tilde{C}^{\sigma} \times\left(S V_{b}^{+}\right)^{u}\left([a, b] \times[a, b], L\left(X, X^{\prime \prime}\right)\right)$ with $\int_{[a, t]} \phi(t, \rho) x d \rho \in X$ for every $t \in[a, b]$ and every $x \in X$, and such that given $f \in \mathbf{K}([\mathbf{a}, \mathbf{b}], \mathbf{X})$, the solution of equation (12) is given by

$$
x_{f}(t)=f(t)-K \int_{[a, b]} \phi(t, s) f(s) d s, \quad t \in[a, b] .
$$

Remark 3.3. By Remark 3.2, Theorems 3.10 and 3.11 hold if we replace $\mathbf{K}([\mathbf{a}, \mathbf{b}], \mathbf{X})$ by $R^{2}([a, b], X)$. These theorems also hold for the Henstock integral with $\mathbf{H}([\mathbf{a}, \mathbf{b}], \mathbf{X})$ instead of $\mathbf{K}([\mathbf{a}, \mathbf{b}], \mathbf{X})$ as well as for the BochnerLebesgue integral replacing $\mathbf{K}([\mathbf{a}, \mathbf{b}], \mathbf{X})$ by $L_{1}([a, b], X)$, where $L_{1}([a, b]$, $X)$ is the space of all equivalence classes of functions $f:[a, b] \rightarrow X$ which are Bochner-Lebesgue integrable with finite integral equipped with the norm $\|f\|_{1}={ }^{L} \int_{[a, b]}\|f(t)\| d t$. 


\subsection{An application.}

Many hypothesis imposed on problems described by integral or differential equations can be weakened by the application of the Kurzweil-Henstock integral setting in solving them. In what follows, we make some comments on the possibility of enlarging the conditions of the functions involved in a certain differential equation.

It is known that the boundary value problem

$$
\left\{\begin{array}{l}
\frac{d^{2} x}{d t^{2}}+A(t) \frac{d x}{d t}+B(t) x=g(t) \\
x(a)=c_{1} \\
x(b)=c_{2}
\end{array}\right.
$$

where the functions involved are continuously differentiable, can be represented by the linear Fredholm integral equation

$$
x(t)-\int_{[a, b]} \alpha(t, s) x(s) d s=f(t), \quad t \in[a, b],
$$

where

$$
f(t)=c_{1}+\int_{[a, t]}(t-s) g(s) d s+\frac{t-a}{b-a}\left[c_{2}-c_{1}-\int_{[a, b]}(b-s) g(s) d s\right]
$$

and

$$
\begin{aligned}
& \alpha(t, s)=\frac{t-b}{b-a}\left\{A(s)-(a-s)\left[A^{\prime}(s)-B(s)\right]\right\}, \quad t>s, \\
& \alpha(t, s)=\frac{t-a}{b-a}\left\{A(s)-(b-s)\left[A^{\prime}(s)-B(s)\right]\right\}, \quad t<s .
\end{aligned}
$$

See $[20]$.

Suppose $g$ is Kurzweil integrable (with, say, $g$ highly oscillating as for instance $g=d G / d t$ where $G(t)=t^{2} \sin t^{-2}, 0<t \leq 1$, and $\left.G(0)=0\right)$. We will prove that $f$ is continuous. Take arbitrary $t \in[a, b]$ and let $h(s)=t-s$, $s \in[a, b]$. Then $h$ is continuous and of bounded variation on $[a, t]$ and Theorem 3.2 implies that $h(\cdot) g(\cdot)$ is Kurzweil integrable with

$$
K \int_{[a, t]} h(s) g(s) d s=\int_{[a, t]} d \tilde{h}(s) g(s),
$$

where $\tilde{h}$ is the indefinite integral of $h$ and hence continuous. The function $\tilde{g}^{\tilde{h}}$ defined by

$$
\tilde{g}^{\tilde{h}}(t)=\int_{[a, t]} d \tilde{h}(s) g(s), \quad t \in[a, b],
$$

is also continuous (for a proof of this fact, see [9, Theorem 1.2]). Thus $f$ is continuous and therefore Riemann integrable. 
We now identify the isomorphic spaces $L(\mathbb{R}, \mathbb{R})$ and $\mathbb{R}$ (see $[24$, pp. 269-270]) and we write $\tilde{C}^{\sigma} \times\left(B V_{b}^{+}\right)^{u}([a, b] \times[a, b], \mathbb{R})$ instead of $\tilde{C}^{\sigma} \times$ $\left(B V_{b}^{+}\right)^{u}\left([a, b] \times[a, b], L\left(\mathbb{R}, \mathbb{R}^{\prime \prime}\right)\right)$.

If $A, A^{\prime}$ and $B$ belong to $B V_{b}^{+}([a, b], \mathbb{R})$, then $\alpha \in \tilde{C}^{\sigma} \times\left(B V_{b}^{+}\right)^{u}([a, b] \times$ $[a, b], \mathbb{R})$. By the analogue of Theorem 3.10 for $R^{2}([a, b], \mathbb{R})$ (see Remarks 3.2 and 3.3), it follows that given $g \in K([a, b], \mathbb{R})$ and functions $A, A^{\prime}, B \in B V_{b}^{+}([a, b], \mathbb{R})$, then either $(25)$ has a unique solution $x_{f}=x_{g} \in$ $R^{2}([a, b], \mathbb{R})$ given by

$$
x_{f}(t)=f(t)-\int_{[a, b]} \phi(t, s) f(s) d s, \quad t \in[a, b],
$$

with unique kernel $\phi \in \tilde{C}^{\sigma} \times\left(B V_{b}^{+}\right)^{u}([a, b] \times[a, b], \mathbb{R})$, or the corresponding homogeneous equation has non-trivial solutions. In this case, (25) has a solution (not necessarily unique) if and only if

$$
\int_{[a, b]}\left[\int_{[a, b]} \alpha(t, s) f(s) d s\right] d z(t)=0,
$$

for every solution $z \in C_{a}([a, b], \mathbb{R})$ of the equation

$$
z(s)-\int_{[a, s]}\left[\int_{[a, b]} \alpha(t, s)^{*} d z(t)\right] d s=0, \quad s \in[a, b] .
$$

All proofs presented here are borrowed from [5].

\section{References}

[1] Alexiewicz, A., Linear functional on Denjoy integrable functions, Colloq. Math. 1 (1948), 289-293.

[2] Bray, H. E., Elementary properties of the Stieltjes integral, Ann. of Math. 20 (191819), 177-186.

[3] Chew, T. S., van-Brunt, B. and Wake, G. C., On retarded functional differential equations and Henstock-Kurzweil integrals, Differential Integral Equations 9(3) (1996), 569-580.

[4] Chew, T. S., van-Brunt, B. and Wake, G. C., First-order partial differential equations and Henstock-Kurzweil integrals, Differential Integral Equations 10(5) (1997), 947960.

[5] Federson, M., Sobre a existência de soluções para Equações Integrais Lineares com respeito a Integrais de Gauge (in Portuguese), Doctor Thesis, Instituto de Matemática e Estatstica da Universidade de São Paulo, São Paulo, 1998.

[6] Federson, M., The fundamental theorem of calculus for multidimensional Banach space-valued Henstock vector integrals, Real Anal. Exchange 25(1) (1999-2000), 469480.

[7] Federson, M., Substitution formulas for the vector integrals of Kurzweil and of Henstock, Math. Bohem. 127(1) (2002), 15-26 (to appear). 
[8] Federson, M., The monotone convergence theorem for multidimensional abstract Kurzweil vector integrals, Czechoslovak Math. J., (to appear in 2002).

[9] Federson, M. and Bianconi, R., Linear integral equations of Volterra concerning the integral of Henstock, Real Anal. Exchange 25(1) (1999-2000), 389-417.

[10] Federson, M. and Bianconi, R., Linear Volterra-Stieltjes integral equations in the sense of the Kurzweil-Henstock integral, Arch. Math. (Basel) 4(37) (2002), 307-328 (to appear).

[11] A. Gilioli, Natural ultrabornological non-complete, normed function spaces, Arch. Math. (Basel) 61 (1993), 465-477.

[12] Gordon, R. A., The Integrals of Lebesgue, Denjoy, Perron and Henstock, Grad. Stud. Math. 4, Amer. Math. Soc., Providence, RI, 1994.

[13] Henstock, R., A Riemann-type integral of Lebesgue power, Canad. J. Math. 20 (1968), 79-87.

[14] Henstock, R., The General Theory of Integration, Oxford Math. Monographs, Clarendon Press, Oxford University Press, New York, 1991.

[15] Hönig, C. S., The Abstract Riemann-Stieltjes Integral and Its Applications to Linear Differential Equations with Generalized Boundary Conditions, Notas do Instituto de Matemática e Estatstica da Universidade de São Paulo Série Mat. 1, Universidade de São Paulo, São Paulo, 1973.

[16] Hönig, C. S., Volterra-Stieltjes Integral Equations. Functional Analytic Methods; Linear Constraints, Math. Stud. 16, North-Holland Publ. Co., Amsterdam, 1975.

[17] Hönig, C. S., Fredholm-Stieltjes integral equations I, Proc. 4th Latin American School of Mathemtics (Lima, 1978), 126-160.

[18] Hönig, C. S., There is no natural Banach space norm on the space of KurzweilHenstock-Denjoy-Perron integrable functions, Sem. Brasileiro Anál. 30 (1989), 387397.

[19] Hönig, C. S., On linear Kurzweil-Henstock integral equations, Sem. Brasileiro Anál. 32 (1990), 283-298.

[20] Jerri, A. J., Introduction to Integral Equations with Applications, Marcel Dekker Inc., New York, Basel, 1985.

[21] Kurzweil, J., Generalized ordinary differential equations and continuous dependence on a parameter, Czechoslovak Math. J., 7(82) (1957), 418-448.

[22] Kurzweil, J., Nichtabsolut Konvergente Integrale, Teubner-Texte Math. 26, Teubner, Leipzig, 1980.

[23] Lee, P. Y., Lanzhou Lectures on Henstock Integration, World Sci., Singapore, 1989.

[24] MacLane, S. and Birkhoff, G., Algebra, The MacMillan Co., London, 1970.

[25] Mc Leod, R. M., The Generalized Riemann Integral, Carus Math. Monographs 20, Math Assoc. America, Washington, 1980.

[26] Muldowney, P., Topics in probability using generalized Riemann integration, Proc. Royal Irish Acad. Sect. A 99(1) 1999, 39-50.

[27] Muldowney, P., Feynman's path integrals and Henstock's non-absolute integration, J. Appl. Anal. 6(1) (2000), 1-24.

[28] Pfeffer, W. F., The Riemann Approach to Integration. Local Geometric Theory, Cambridge Tracts in Math. 9, Cambridge University Press, Cambridge, 1993.

[29] Schwabik, S., Abstract Perron-Stieltjes integral, Math. Bohem. 121(4), (1996), 425447.

[30] Schwabik, S., Linear Stieltjes integral equations in Banach spaces, Math. Bohem. 124(4) (1999), 433-457.

[31] Tvrdý, M., Linear integral equations in the space of regulated functions, Math. Bohem. 123(2) (1998), 177-212. 
M. FEDERSON

Instituto DE CiênCIAs MATEmÁticAs

E DE ComputaÇÃO

Universidade de SÃo Paulo

CP 688, SÃo Carlos

SP 13560-970, BRAZIL

E-MAIL: FEDERSON@ICMC.SC.USP.BR
R. BIANCONI

Instituto DE MATEMÁticA

e Estatstica

Universidade de SÃo Paulo

CP 66281, SÃo Paulo

SP 05315-970, BRAZIL

E-MAIL: BIANCONI@IME.USP.BR 